\title{
DIFERENCIAS SOCIOECONÓMICAS EN LA POSTULACIÓN A LAS UNIVERSIDADES CHILENAS: EL ROL DE FACTORES ACADÉMICOS Y NO ACADÉMICOS ${ }^{1}$
}

\author{
Andrea Canales ${ }^{2}$
}

\begin{abstract}
RESUMEN
Numerosas investigaciones han dado cuenta de la estrecha relación entre las desigualdades socioeconómicas en el acceso a la educación universitaria en Chile y la preparación/resultados académicos de los postulantes. La abundante evidencia acerca de los factores académicos contrasta con los escasos estudios centrados en la influencia de factores no académicos en el acceso al sistema universitario. Utilizando los registros administrativos del proceso de admisión universitario $2013(n=264.023)$, se analizan los factores académicos y no académicos que influyen en la decisión de postular a las universidades. Los resultados confirman que en dicha decisión los resultados obtenidos en las pruebas de selección universitaria (PSU), así como también, la educación parental y las expectativas educativas de los postulantes son factores influyentes. Específicamente, se constató que el origen socioeconómico opera en la decisión de postulación a partir de la evaluación que los postulantes hacen de sus capacidades y expectativas de éxito en el sistema universitario.
\end{abstract}

Palabras clave: diferencias socioeconómicas, acceso, expectativas educativas, postulación, universidades

\section{SOCIOECONOMIC DIFFERENCES IN THE APPLICATION TO CHILEAN UNIVERSITIES: THE ROLE OF ACADEMIC AND NON-ACADEMIC FACTORS}

\section{ABSTRACT}

Numerous studies have shown that socioeconomic inequalities, manifested through differences in student's prior academic preparation or achievement, strongly influence access to Chilean universities. To date, the research has focused mainly on the influence of academic factors on inequalities in access, paying little attention to the role of non-academic factors in this process. Administrative records of the university admission process in 2013 ( $n=$ 264,023) were used to study how academic and non-academic factors influence the decision to apply to universities. The findings confirm that the decision to apply to the college admission process is strongly associated with the scores on the university admission tests (PSU), as well as parental education and the applicants' educational expectations. In particular, the findings suggest that students' socioeconomic backgrounds influence their decision to apply to universities, arising from their evaluation of their own abilities and expectations of success in the university system.

Keywords: socioeconomic differences, access, educational expectations, application, universities.

1 Este trabajo fue realizado como parte del proyecto Dicyt Regular 031411CH "Desigualdades socioeconómicas en la transición a la educación superior: su relación con la preparación académica y decisiones educativas de los postulantes", financiado por la Vicerrectoría de Investigación Desarrollo e Innovación (VRIDEI) de la Universidad de Santiago de Chile durante los años 2014-2015. La autora, en ese entonces, se desempeñaba como profesora asistente de la Universidad de Santiago de Chile.

2 Instituto de Sociología, Pontificia Universidad Católica de Chile. Santiago, Chile, acanales@ uc.cl 


\section{Introducción}

En las últimas décadas, la educación superior chilena ha experimentado cambios significativos asociados especialmente a la expansión y diversificación de su oferta educativa. De acuerdo con cifras del Ministerio de Educación de Chile, Mineduc (2012), la cobertura neta ${ }^{3}$ del sistema terciario creció significativamente en la última década, llegando a un 36\% en el año 2011. Tasas más altas de participación en el sistema no implican necesariamente una mayor igualdad en el acceso. La evidencia empírica indica que las diferencias socioeconómicas en el acceso a la educación superior siguen persistiendo, en particular, en el acceso al sistema universitario (OECD, 2012).

Para explicar estas desigualdades socioeconómicas, la literatura nacional ha centrado su atención en temas académicos y económicos. Por una parte, las investigaciones han dado cuenta de la estrecha relación entre origen socioeconómico y preparación/resultados académicos de los postulantes. Entre los factores que contribuyen mayormente a explicar las desigualdades en el acceso a la educación superior en Chile están las diferencias significativas en el rendimiento en las pruebas de selección universitaria y preparación académica previa de los postulantes (Donoso y Cancino, 2007; García Huidobro y Bellei, 2003; Hernández y Paredes, 2007; Manzi, 2006; Muñoz y Redondo, 2013; Silva y Koljatic, 2010; Valdivieso, Antivilo y Barrios, 2006). Por otra parte, los estudios han señalado que los altos costos asociados a la educación superior y las alternativas de financiamiento existentes son factores que limitan las posibilidades de elección e ingreso de estudiantes de grupos socioeconómicos menos aventajados al sistema (Manzi, 2006; Rodríguez, 2012).

La abundante evidencia acerca de factores académicos y su relación con las desigualdades socioeconómicas en el acceso a la educación superior en Chile contrasta con los escasos estudios respecto del rol de los factores no académicos -que no sean económicos- en este proceso. En el ámbito internacional, la literatura muestra que

3 La tasa de cobertura neta se define como el total de personas en educación superior entre 18 y 24 años sobre el total de personas del mismo rango de edad. 
las desigualdades socioeconómicas en el acceso al sistema terciario se vinculan tanto con diferencias en las habilidades académicas, como también con aspectos motivacionales, expectativas o aspiraciones educativas y ocupacionales de los estudiantes, entre otros aspectos (Breen \& Goldthorpe, 1997; Morgan, Leenman, Todd \& Weeden, 2013; Shavit, Arum, Gamoran \& Menahem, 2007).

Investigaciones sociológicas ligadas a los modelos de acción racional plantean que las desigualdades socioeconómicas ${ }^{4}$ en el acceso a la educación superior se asocian tanto con los resultados académicos de los estudiantes, como también, con la evaluación que estos hacen de los beneficios, costos y sus probabilidades de éxito en el sistema educativo (Bernardi \& Cebolla, 2014; Breen, 1999; Breen \& Goldthorpe, 1997; Erikson \& Jonsson, 1996; Jackson, Erikson, Goldthorpe \& Meir, 2007). Esta literatura distingue dos tipos de efectos del origen socioeconómico. Por una parte, la asociación entre origen socioeconómico ${ }^{5}$ y resultados educativos del estudiante da cuenta de los efectos primarios del origen social. Por otra, la asociación entre origen socioeconómico y probabilidad subjetiva de éxito en el sistema educativo corresponde a los efectos secundarios del origen social. Este último aspecto da cuenta de las diferencias socioeconómicas en la evaluación que los jóvenes y sus familias realizan de sus habilidades a la hora de decidir continuar estudios en un nivel superior (Breen, 1999). La evidencia empírica ${ }^{6}$ muestra que jóvenes de clases sociales más acomodadas tienden a sobrestimar sus opciones de éxito en comparación con los jóvenes de clases menos aventajadas, aun cuando ambos grupos tengan rendimientos o resultados académicos similares. Lo anterior explica por qué jóvenes de clases más aventajadas deciden en mayor proporción continuar estudios superiores (Breen, 1999; Breen \& Goldthorpe, 1997; Erikson

4 Raymon Boudon (1974) es considerado como el punto de partida de los modelos de acción racional en sociología ligados al estudio de las desigualdades educativas. El autor introdujo la distinción entre efectos primarios y secundarios del origen social para analizar las desigualdades educativas en Francia.

5 La mayoría de estos estudios remiten principalmente a diferencias de clase social en los logros educativos.

6 Iatridis y Fousiani (2009) encontraron en un estudio experimental que el éxito académico para estudiantes de clase alta y el fracaso académico para alumnos de clases bajas se atribuía a la habilidad académica, mientras que el fracaso académico entre estudiantes de estrato alto y el éxito académico entre estudiantes de estratos bajos tendía a atribuirse a esfuerzo. 
\& Jonsson, 1996; Roksa, Grodsky, Arum \& Gamoran, 2007; Shavit et al., 2007).

De forma complementaria, estudios ligados al modelo de Wisconsin (Sewell, Haller \& Portes, 1969) -que plantea que factores psicosociales influyen en los logros ocupacionales y educativos de los individuos- han encontrado que las expectativas ${ }^{7}$ educativas de los estudiantes tienen un rol importante en las desigualdades socioeconómicas en el acceso a la educación superior. En particular, los investigadores han señalado que existe una estrecha relación entre el origen socioeconómico y las expectativas educacionales de los jóvenes (Bozick, Alexander, Entwisle, Dauber \& Kerr, 2010; Lareau $\&$ Weininger, 2003; Morgan, 2002; Morgan et al., 2013; Reynolds \& Johnson, 2011). Así, jóvenes de estratos sociales más aventajados tienen, en general, expectativas más arraigadas y precisas ${ }^{8}$ acerca de su futuro educativo, lo que explicaría sus mayores probabilidades de acceder y mantenerse en el sistema de educación superior (Bozick et al., 2010; Morgan et al., 2013). Las diferencias socioeconómicas en términos de expectativas se explican, en parte, por los rendimientos y/o desempeños académicos de los estudiantes (Andrew \& Hauser, 2012; Buchman \& Park, 2009; Jerrim, Chmielewski \& Parker, 2015; OECD, 2010, 2012). Los jóvenes definen sus planes futuros educativos sobre la base de la evaluación que realizan de sus habilidades académicas.

La influencia del entorno familiar y social más directo del estudiante es otro de los factores que ayuda a explicar las diferencias socioeconómicas en las expectativas educativas. Las aspiraciones y

7 En los últimos años, se observa una serie de estudios que han abordado el tema de las aspiraciones y expectativas sobre educación superior en Chile (ver Castillo y Cabezas, 2010; Jiménez y Lagos, 2011; Sepúlveda y Valdebenito, 2014, entre otros). La mayoría de estos estudios ha trabajado con metodologías cualitativas.

8 En las últimas décadas, se ha observado un cambio en el patrón de expectativas y aspiraciones educativas de la población en general (Reynolds, 2006). Jóvenes de todos los orígenes sociales aspiran a continuar estudios superiores (Castillo y Cabezas, 2010; Jiménez y Lagos, 2011; Reynolds \& Johnson, 2011; Sepúlveda y Valdebenito, 2014). En este contexto de expectativas y aspiraciones generalizadas, las diferencias socioeconómicas se han trasladado al plano de la concreción de estas expectativas educativas. Para los alumnos de orígenes no aventajados existiría un ajuste más directo de sus aspiraciones sobre la base de los requerimientos económicos y/o las exigencias académicas que estas demandan (Sepúlveda y Valdebenito, 2014). 
proyecciones que padres ${ }^{9}$, pares y profesores tienen sobre los jóvenes influyen en la forma en la que estos definen sus aspiraciones o proyecciones respecto de su futuro educativo y ocupacional (Sewell et al., 1969). Los jóvenes de estratos sociales más aventajados reciben el apoyo y aliento constante de sus padres respecto de sus planes futuros, aun en circunstancias en que sus desempeños académicos no sean destacados (Bernardi \& Cebolla, 2014). La evidencia muestra que padres con altas expectativas se involucran activamente o directamente en el proceso educativo de sus hijos desde edades tempranas (Coleman, 1988; Hossler \& Stage, 1992; McDonough, 1997). Por el contrario, los jóvenes de origen social menos acomodado no reciben claras señales de sus padres y otros seres significativos respecto de sus capacidades y lo que se espera de ellos.

Como se puede apreciar, la literatura internacional ha analizado las desigualdades socioeconómicas en el acceso a la educación superior desde una perspectiva multidimensional. El presente artículo pretende avanzar en esta misma dirección. Usando registros administrativos del proceso de admisión a las universidades chilenas del año 2013 $(n=264.023)$, este estudio tiene por objetivo analizar el rol de los factores académicos y no académicos en la decisión ${ }^{10}$ de postular a las universidades chilenas.

Específicamente, esta investigación se centra en analizar:

a. la influencia del rendimiento en enseñanza media y puntajes en pruebas de selección universitaria en la decisión de postulación;

b. la influencia del origen socioeconómico en la decisión de postulación;

c. la influencia de las expectativas educativas en la decisión de postulación y;

d. cómo los rendimientos académicos, expectativas y decisiones

9 La literatura agrega que los jóvenes también influyen en las expectativas que los otros tienen sobre ellos, a partir de su desempeño académico escolar.

10 Este estudio analiza el proceso de postulación a las universidades adscritas al sistema único de admisión universitario administrado por Departamento de Evaluación, Medición y Registro Educacional, Demre. Este sistema congrega a 25 universidades del Consejo de Rectores (CRUCH) y ocho universidades privadas del país. 
educativas de los postulantes se relacionan con las diferencias socioeconómicas en la postulación a las universidades chilenas.

A continuación, se describen en detalle las fuentes de información, variables y métodos utilizados en este estudio.

\section{Datos, variables y métodos}

\subsection{Datos}

Este artículo utiliza registros administrativos de los postulantes que participaron del proceso de admisión a las universidades ${ }^{11}$ chilenas durante el año 2013 como principal fuente de información. Estos registros entregan información detallada sobre las distintas etapas que contempla el proceso de admisión (inscripción, rendición de pruebas de selección, postulación a las universidades/carreras, selección y matrícula), como también, de las características sociodemográficas de los postulantes y sus resultados educativos. Estos registros son administrados por el Departamento de Medición, Admisión y Registro de la Universidad de Chile (Demre) ${ }^{12}$. Este es el organismo que administra y centraliza el proceso de admisión y selección para el ingreso a las 25 universidades que conforman el Consejo de Rectores (CRUCH), más ocho universidades privadas que se adscribieron a este proceso a partir del año 2011. Estas universidades conforman el grupo de instituciones más selectivas del sistema de educación superior chileno, y concentran alrededor de un $40 \%$ de la matrícula total del sistema.

El universo con el que trabaja esta investigación corresponde a 264.023 individuos, que representa un 96,8\% del universo total de

11 La mayor parte de las universidades creadas con posterioridad al año 1981 aplican un sistema de admisión distinto al sistema de admisión coordinado por Demre. Estas instituciones cuentan con requisitos y calendarios propios de admisión, los que no están unificados en un sistema de admisión común.

12 Se extienden los agradecimientos al Departamento de Medición, Registro y Evaluación (Demre) de la Universidad de Chile, a la Unidad de Estadísticas del Centro de Estudios del Ministerio de Educación, al Registro Académico de la Universidad de Santiago de Chile (USACH) y a la Agencia de Calidad de la Educación por los antecedentes entregados, que permitieron llevar a cabo este estudio. 
personas que se inscribieron y participaron del proceso de admisión a las universidades chilenas en el año 2013.

Junto con los datos del Demre, se utilizó también información de los cuestionarios del Sistema Nacional de Evaluación de Aprendizajes (Simce) ${ }^{13}$, que fueron aplicados a estudiantes de $2^{\circ}$ medio del año 2010. Estos cuestionarios entregan información retrospectiva acerca de las expectativas educativas de los postulantes, mientras estos se encontraban cursando dicho nivel. Se empalmó la información Simce con los registros del Demre. El empalme de ambas bases de datos mostró que alrededor de un $40 \%$ de los inscritos en el proceso de admisión 2013, contaba con información en los cuestionarios Simce.

\subsection{Variables}

\subsubsection{Variable dependiente}

La variable dependiente que se analiza en este estudio corresponde a una variable dicotómica, que da cuenta de la probabilidad de postular al sistema de admisión universitario chileno. Se entiende la postulación ${ }^{14}$ a dicho sistema como un proxy o indicador del ingreso a la educación universitaria en Chile.

\subsubsection{Variables independientes}

a. Variables académicas

Se utilizaron tres variables para dar cuenta del rendimiento y/o preparación académica de los postulantes. Las tres provienen de los registros del Demre y corresponden a requisitos de admisión al sistema universitario.

13 El empalme de los datos Demre-Simce fue realizado por la Unidad de Estadísticas del Centro de Estudios del Ministerio de Educación. El Demre autorizó a esta unidad para realizar este procedimiento, con el fin de que se protegiera la anonimidad de los sujetos.

14 Es importante destacar que la decisión de postular al sistema de admisión universitario compete directamente al postulante ( $\mathrm{y}$ a sus familias). Este proceso no involucra directamente a las instituciones de educación superior, a diferencia de lo que ocurre con el acceso al sistema. 
La primera de ellas es el puntaje promedio en las pruebas obligatorias de selección universitaria (PSU) de Matemática y Lenguaje. La segunda corresponde al promedio de notas obtenido durante la enseñanza media por los postulantes. Específicamente, se utilizó el puntaje que estandariza este promedio de notas para hacerlos comparables entre sí (puntaje NEM). Finalmente, la tercera variable concierne al puntaje ranking asignado por la unidad educativa donde el postulante cursó sus estudios de enseñanza media. Esta medida se comenzó a utilizar en el proceso de admisión universitario del año 2013 y expresa la posición relativa del estudiante en su escuela, tomando como referencia el desempeño académico de los alumnos de las últimas tres generaciones en la unidad educativa. La Tabla 1 presenta la descripción de las tres variables académicas utilizadas en este estudio.

Tabla 1. Descriptivos para variables académicas

\begin{tabular}{l|c|c|c|c|c}
\hline Variables & N & Media & $\begin{array}{c}\text { Desviación } \\
\text { estándar }\end{array}$ & Mínimo & Máximo \\
\hline $\begin{array}{l}\text { Puntaje notas enseñanza media } \\
\text { (NEM) }\end{array}$ & 240.185 & 539,6 & 99,9 & 208 & 826 \\
\hline Puntaje promedio PSU & 230.112 & 500,7 & 101,9 & 192,5 & 837,5 \\
\hline Puntaje ranking & 240.185 & 558,3 & 120,9 & 208 & 850 \\
\hline
\end{tabular}

b. Variable expectativas educativas

En el cuestionario Simce aplicado a alumnos de $2^{\circ}$ medio 2010, se les consultó acerca de sus proyecciones o aspiraciones educativas. Se utilizó la pregunta relacionada con el nivel educativo que el postulante esperaba completar en ese entonces a modo de indicador de sus expectativas educativas ${ }^{15}$.

15 Diversas investigaciones han señalado que las expectativas de los jóvenes en etapas finales de su educación obligatoria o secundaria es un buen predictor de la decisión que tomarán en relación con su futuro educacional. 


\section{c. Variables de origen socioeconómico ${ }^{16}$}

Se utilizaron tres variables relativas a la educación y ocupación parental para dar cuenta del origen socioeconómico del postulante. Estas provienen de la base de datos Demre y fueron reportadas ${ }^{17}$ por los propios postulantes al momento de inscribirse en el proceso de admisión universitario del año 2013.

Para dar cuenta del nivel educacional ${ }^{18}$ de la familia del postulante, se consideró la educación de la madre y del padre del postulante por separado. Ambas variables fueron reagrupadas, siguiendo la Clasificación Internacional Normalizada de Educación (ISCED) de la Unesco. Esta clasificación da cuenta del máximo nivel educacional alcanzado por los individuos. Las categorías que conforman esta clasificación educativa son: sin estudios, educación primaria, educación secundaria incompleta, educación secundaria completa, educación terciaria de ciclo corto ${ }^{19}$ y educación terciaria de ciclo largo ${ }^{20}$.

16 En este estudio, se consideran solo variables de origen socioeconómico a nivel individual. No se utilizan variables proxy de origen social, tales como dependencia del establecimiento educacional o modalidad de estudios cursada en enseñanza media, ya que estas dan cuenta del origen social del estudiantado a nivel agregado.

17 La literatura internacional señala que los reportes de los estudiantes sobre su situación socioeconómica se ajustan, en buena medida, con los que sus padres entregan en relación con la realidad socioeconómica de la familia. La ocupación parental es la categoría donde existe mayor concordancia entre padres e hijos; existiendo concordancia también en lo que refiere a la educación de los padres.

18 Existe consenso en la literatura respecto de la centralidad de la educación de los padres en los resultados educativos de los estudiantes, especialmente en edades tempranas del ciclo escolar. En Chile, a menudo se ha utilizado una medida que combina la educación de ambos padres o, alternativamente, solo la educación de la madre del estudiante para ver su relación con los resultados educativos de este. Dado que este artículo se centra en analizar una decisión educativa (no un resultado educativo) que los jóvenes deben tomar una vez que finalizan sus estudios secundarios, se estimó pertinente indagar en la influencia que ambos padres pudieran tener en esa decisión.

19 Refiere a estudios técnicos y/o técnicos profesionales de nivel superior de duración menor a tres años.

20 Refiere principalmente a estudios universitarios y/o carreras profesionales de duración mayor a tres años. 
A partir de información acerca de la ocupación del padre ${ }^{21}$ del postulante, se construyó la variable clase social de origen ${ }^{22}$ del mismo. Los siguientes parámetros se usaron para ello:

- situación ocupacional del padre: se consideró solo a personas económicamente activas;

- la ocupación específica del padre;

- la rama de actividad en la que el padre se encontraba ocupado $\mathrm{y}$

- el estatus ocupacional del padre (empleador o empleado).

Para clasificar las clases sociales se empleó la metodología seguida por Torche (2005), quien adaptó para Chile el esquema de clases sociales de Erikson, Goldthorpe y Portocarrero (EGP). Este ha sido ampliamente utilizado en estudios de desigualdad educativa y movilidad social a nivel comparado. Las categorías que lo conforman corresponden a las siguientes: clase de servicios (profesionales universitarios, gerentes, dueños de grandes empresas), clase de rutina no manual, pequeña burguesía, trabajadores independientes, trabajadores manuales calificados, trabajadores manuales no calificados, pequeños propietarios agrícolas y trabajadores agrícolas.

La Tabla 2 presenta la descripción de las variables de origen socioeconómico y expectativas educativas utilizadas en este estudio.

21 A diferencia de la metodología seguida por Torche (2005), no fue posible en este estudio utilizar información sobre el tipo de contrato que tenía el padre del postulante en la construcción de la variable clase social. El Demre no recopila esta información.

22 Se utiliza la clase social del padre del estudiante como un indicador de la posición relativa de la familia del estudiante en la estructura económica. En este estudio, no se incluye la variable clase social de la madre del postulante, dado que un $45 \%$ de ellas fueron reportadas como dueñas de casa. De acuerdo con la clasificación EGP, no es posible asignar una clase social a personas que se encuentran inactivas económicamente. 
Tabla 2. Descriptivos para variables de origen socioeconómico y expectativas educativas

\begin{tabular}{|c|c|c|}
\hline Variables & N & $\%$ \\
\hline Clase social padre & 264,023 & \\
\hline Clase de servicios & & 0,17 \\
\hline Clase de rutina no manual & & 0,15 \\
\hline Pequeña burguesía & & 0,05 \\
\hline Trabajadores independientes & & 0,02 \\
\hline Trabajadores manuales calificados & & 0,10 \\
\hline Trabajadores manuales no calificados & & 0,15 \\
\hline Pequeños propietarios agrícolas & & 0,01 \\
\hline Trabajadores agrícolas & & 0,06 \\
\hline Sin información & & 0,29 \\
\hline Nivel educacional padre & 264,023 & \\
\hline Sin estudios & & 0,01 \\
\hline Educación primaria & & 0,17 \\
\hline Educación secundaria incompleta & & 0,12 \\
\hline Educación secundaria completa & & 0,30 \\
\hline Educación terciaria de ciclo corto & & 0,09 \\
\hline Educación terciaria de ciclo largo & & 0,16 \\
\hline Sin información & & 0,17 \\
\hline Nivel educacional madre & 264,023 & \\
\hline Sin estudios & & 0,01 \\
\hline Educación primaria & & 0,18 \\
\hline Educación secundaria incompleta & & 0,13 \\
\hline Educación secundaria completa & & 0,35 \\
\hline Educación terciaria de ciclo corto & & 0,12 \\
\hline Educación terciaria de ciclo largo & & 0,13 \\
\hline Sin información & & 0,08 \\
\hline Expectativas educativas & 94,220 & \\
\hline No cree que llegue a completar $4^{\circ}$ medio & & 0,01 \\
\hline Cuarto medio & & 0,02 \\
\hline Carrera en instituto prof. 0 centro de formación técnica & & 0,06 \\
\hline Carrera universitaria & & 0,28 \\
\hline Sin información & & 0,64 \\
\hline
\end{tabular}




\section{Métodos}

Dos tipos de análisis se emplearon en este artículo. En primera instancia, se desarrollan los análisis descriptivos y de asociación para las variables de interés de este estudio. Luego, se emplean modelos de regresión logística para analizar la relación de los factores académicos y no académicos sobre la probabilidad de postular al sistema de admisión universitario chileno. La especificación del modelo utilizado se expone en la siguiente ecuación:

$$
\log (\pi /(\pi-1))=\beta_{0}+\beta_{1} x_{1}+\beta_{2} x_{2}+\beta_{3} x_{3}
$$

Donde $\pi$ es la probabilidad que $Y=1$, es decir, que el estudiante postule al sistema único de admisión universitario. La postulación al sistema de admisión se entiende como la función de: un intercepto $\left(\beta_{0}\right)$, donde $\left({ }_{\mathrm{X} 1}\right)$ representa a cualquier factor de tipo académico; $\left({ }_{\mathrm{X} 2}\right)$ representa a cualquier variable de origen socioeconómico, y $(x)$ representa a un factor relacionado con expectativas educativas. A través de métodos de imputación múltiple $(\mathrm{MI})^{23}$, se atribuyeron los casos perdidos a las variables con información incompleta.

\section{Resultados}

\subsection{Antecedentes descriptivos}

De acuerdo con antecedentes del Demre, en el proceso de admisión a las universidades chilenas del año 2013 participó un total de 272.666 postulantes. De este total de inscritos, un $87 \%$ rindió las pruebas de selección universitaria (PSU). En relación con los resultados en las pruebas de selección, las cifras revelan que alrededor de un 60\% de quienes rindieron estas pruebas obtuvo un puntaje igual o superior a 475 puntos $^{24}$; este puntaje corresponde al puntaje mínimo para poder

23 Los métodos de imputación múltiple se emplearon para imputar solo los valores perdidos de la variable expectativas educativas; la cual contaba con un $60 \%$ de casos perdidos.

24 Si bien el puntaje mínimo requerido para postular al sistema único de admisión universitario es de 475 puntos, se observa una significativa variación en los requisitos de postulación por universidad en el sistema de admisión. Una breve revisión de estos requisitos muestra, por ejemplo, que las Universidades de Chile y la P. Universidad Católica exigen un puntaje mínimo ponderado de 600 puntos para postular a sus carreras; mientras que algunas universidades regionales pertenecientes al Consejo de Rectores, exigen en ciertas carreras puntajes incluso inferiores a 475 puntos o solo exigen rendición de la PSU. 
postular ${ }^{25}$ al proceso de admisión universitario. Respecto del proceso de postulación, los antecedentes indican que solo un 44\% de quienes participaron postuló a alguna de las carreras e instituciones adscritas al proceso de admisión universitario.

En la Tabla 3, se presenta la distribución de las tasas de postulación ${ }^{26}$ al sistema de admisión universitario junto con los puntajes promedio ${ }^{27}$ en las pruebas de selección universitaria para cada una de las variables de interés. Junto con lo anterior, la Tabla 3 también entrega estadísticos de correlación entre los puntajes promedio PSU y las variables de interés (rho), y test de correlación entre las tasas de postulación al sistema de admisión universitario y las variables independientes usadas en este estudio $\left(X^{2}\right)$.

Confirmando lo planteado en investigaciones previas, se observa una asociación positiva entre los puntajes en las pruebas de selección universitaria y las tasas de postulación al sistema de admisión (Valdivieso et al., 2006). Los jóvenes que obtienen puntajes más altos en estas presentan mayores tasas de postulación al sistema de admisión universitario. El proceso de postulación supone que los jóvenes comparen sus resultados PSU con los puntajes de corte ${ }^{28}$ de las carreras e instituciones de su preferencia. En el caso de que sus puntajes no cumplan con los requisitos establecidos por estas, los postulantes deben reevaluar sus opciones y postular a otras carreras o instituciones en las que tengan más oportunidades de ser aceptados o seleccionados. Los jóvenes tienen un máximo de 10 opciones de postulación al sistema de admisión universitario.

25 Aun cuando alcanzaron un puntaje suficiente para hacerlo, un grupo significativo de postulantes decidió no postular al sistema de admisión. Solo un $63 \%$ de los postulantes con puntajes iguales o superiores a 475 puntos efectivamente postuló al sistema de admisión universitario.

26 En el cálculo de las tasas de postulación se consideró la primera preferencia que los postulantes reportaron en su proceso de postulación, independiente de la carrera e institución a la que hayan postulado.

27 El puntaje promedio PSU se obtuvo sumando los puntajes de las pruebas obligatorias de selección universitaria de matemáticas y lenguaje obtenidos por cada estudiante.

28 Los puntajes de corte refieren al puntaje del último seleccionado en la carrera en el proceso de admisión del año anterior. 
Un análisis por quintil de puntaje PSU nos muestra una variación significativa en las tasas de postulación al sistema. Como se observa, solo un $47 \%$ de aquellos que obtuvieron entre 475 y 525 puntos promedio en la PSU postuló al sistema de admisión ${ }^{29}$. Estas cifras ascienden a un $75 \%$ para los que obtuvieron puntajes en el cuarto quintil de puntajes (entre 536 y 589 puntos), y a un 92\% para los que obtuvieron puntajes sobre los 590 puntos (quintil más alto de puntajes). Por otra parte, los antecedentes confirman una asociación positiva entre las tasas de postulación y las variables de desempeño académico en la enseñanza media. Mejores notas en la enseñanza media están importantemente correlacionadas con mayores puntajes en las pruebas de selección universitaria (rho $=0,57$ ). Asimismo, mejores calificaciones (notas y puntaje ranking asociado) en la enseñanza media, se asocian con mejores tasas de postulación al sistema de admisión universitario.

Por otra parte, se observa que las características socioeconómicas de los postulantes también se correlacionan con sus logros educativos. La evidencia muestra una asociación positiva entre el nivel educacional de los padres -especialmente de la madre- y los puntajes PSU del postulante. A medida que aumenta el nivel educacional de ambos padres del postulante, los puntajes PSU crecen. Las mayores diferencias de puntajes se observan entre postulantes cuyos padres tienen estudios universitarios y postulantes con padres con educación primaria incompleta o sin estudios. Una brecha socioeconómica también se observa en relación con las tasas de postulación al sistema de admisión universitario. Postulantes con padres con estudios universitarios son el grupo con mayor presencia entre los que postulan al sistema de admisión universitario. Respecto de la clase social de origen, los antecedentes también confirman una asociación significativa con los puntajes en las pruebas de selección universitaria, aunque esta asociación es más bien moderada. Postulantes cuyo padre desempeña una ocupación gerencial y/o profesional obtienen en promedio 100 puntos más en las pruebas de selección universitaria que aquellos

29 Debe señalarse que, dada la información disponible, no es posible saber si los jóvenes postularon a instituciones universitarias que no son parte del sistema único de admisión universitario o, por otra parte, postularon a instituciones de educación superior no universitarias. 
cuyos padres desempeñan trabajos agrícolas o realizan una ocupación manual no calificada. Con respecto a los índices de postulación, la evidencia confirma que postulantes con padres con cargos gerenciales o profesionales son quienes predominan entre aquellos que postulan al sistema de admisión universitario.

Finalmente, respecto de las expectativas educativas, se observa que estas se correlacionan positivamente con los resultados educativos de los postulantes. Expectativas educativas más altas -que corresponden a expectativas universitarias- se asocian con mejores puntajes en las pruebas de selección universitaria y, por otra parte, con mayores tasas de postulación al sistema de admisión universitario.

En resumen, los antecedentes presentados entregan evidencia para sustentar la hipótesis de asociación entre las variables independientes y la variable dependiente de este estudio. En la siguiente sección, se estimará la estabilidad de estas asociaciones, una vez que se incorporen los predictores de interés y controles a los modelos de regresión logística.

Tabla 3. Puntajes promedio PSU y porcentaje que postula al sistema de admisión, de acuerdo con variables académicas, socioeconómicas y expectativas educativas

\begin{tabular}{|c|c|c|}
\hline Variables & $\begin{array}{c}\text { Puntajes promedio PSU } \\
\text { (desv,std,) }\end{array}$ & $\%$ que Postula \\
\hline \multicolumn{3}{|l|}{ Puntaje NEM } \\
\hline Primer quintil & $440,2(74,2)$ & 0,23 \\
\hline Segundo quintil & $464,7(80,9)$ & 0,34 \\
\hline Tercer quintil & $499,8(87,8)$ & 0,50 \\
\hline Cuarto quintil & $529,4(92,1)$ & 0,62 \\
\hline \multirow[t]{2}{*}{ Quinto quintil } & $599,1(95,0)$ & 0,81 \\
\hline & Rho $=0,57, p<0,001$ & $x 2(4)=3,4 e+04, p<0,001$ \\
\hline \multicolumn{3}{|l|}{ Puntaje ranking } \\
\hline Primer quintil & $440,2(59,3)$ & 0,23 \\
\hline Segundo quintil & $465,6(56,1)$ & 0,35 \\
\hline Tercer quintil & $497,1(54,2)$ & 0,49 \\
\hline Cuarto quintil & $533,3(53,5)$ & 0,63 \\
\hline \multirow[t]{2}{*}{ Quinto quintil } & $577,6(63,3)$ & 0,74 \\
\hline & Rho $=0,54, p<0,001$ & $x 2(4)=3,5 e+04, p<0,001$ \\
\hline
\end{tabular}


144 DIFERENCIAS SOCIOECONÓMICAS EN LA POSTULACION A LAS UNIVERSIDADES CHILENAS: EL ROL DE FACTORES ACADÉMICOS Y NO ACADÉMICOS - A. Canales

\begin{tabular}{|c|c|c|}
\hline \multicolumn{3}{|l|}{ Promedio PSU } \\
\hline Primer quintil (192.5-410.) & & 0,05 \\
\hline Segundo quintil (410.5-474.) & & 0,17 \\
\hline Tercer quintil (475-525.5) & & 0,47 \\
\hline Cuarto quintil (526-589) & & 0,75 \\
\hline \multirow[t]{2}{*}{ Quinto quintil (589.5-850) } & & 0,92 \\
\hline & & $x 2(4)=1,0 e+04, p<0,001$ \\
\hline \multicolumn{3}{|l|}{ Clase social padre } \\
\hline Clase de servicio & $566,9(100,2)$ & 0,68 \\
\hline Clase de rutina no manual & $505,8(92,1)$ & 0,48 \\
\hline Pequeña burguesía & $527,3(95,4)$ & 0,56 \\
\hline Trabajadores independiente & $491,6(91,7)$ & 0,39 \\
\hline Trabajadores manuales calificados & $479,9(88,8)$ & 0,38 \\
\hline Trabajadores manuales no calificados & $467,4(88,8)$ & 0,32 \\
\hline Pequeños propietarios agrícolas & $532,4(104,6)$ & 0,58 \\
\hline \multirow[t]{2}{*}{ Trabajadores agrícolas } & $455,9(87,3)$ & 0,29 \\
\hline & Rho $=-0,25, p<0,001$ & $X 2(4)=1,6 e+04, p<0,001$ \\
\hline \multicolumn{3}{|l|}{ Nivel educacional padre } \\
\hline Sin estudios & $439,6(92,9)$ & 0,22 \\
\hline Ed. Primaria & $452,1(87,3)$ & 0,26 \\
\hline Ed. Secundaria incompleta & $469,3(87,6)$ & 0,33 \\
\hline Ed. Secundaria completa & $491,2(90,4)$ & 0,42 \\
\hline Ed. Terciaria de ciclo corto & $535,5(89,9)$ & 0,60 \\
\hline \multirow[t]{2}{*}{ Ed. Terciaria de ciclo largo } & $579,9(94,9)$ & 0,72 \\
\hline & Rho $=0,33, p<0,001$ & $x 2(4)=2,3 e+04, p<0,001$ \\
\hline \multicolumn{3}{|l|}{ Nivel educacional madre } \\
\hline Sin estudios & $434,8(95,7)$ & 0,20 \\
\hline Ed. Primaria & $450,1(86,9)$ & 0,25 \\
\hline Ed. Secundaria incompleta & $465,7(86,8)$ & 0,31 \\
\hline Ed. Secundaria completa & $495,6(91,1)$ & 0,44 \\
\hline Ed. Terciaria de ciclo corto & $540,3(91,8)$ & 0,62 \\
\hline \multirow[t]{2}{*}{ Ed. Terciaria de ciclo largo } & $582,2(95,9)$ & 0,72 \\
\hline & Rho $=0,57, p<0,001$ & $x 2(4)=2,5 e+04, p<0,001$ \\
\hline \multicolumn{3}{|l|}{ Expectativas educativas } \\
\hline No cree que llegue a completar $4^{\circ}$ medio & $432,8(114,2)$ & 0,22 \\
\hline Cuarto medio & $416,9(80,9)$ & 0,12 \\
\hline $\begin{array}{l}\text { Carrera en instituto prof. o centro de } \\
\text { formación técnica }\end{array}$ & $435,3(75,3)$ & 0,15 \\
\hline \multirow[t]{2}{*}{ Carrera universitaria } & $518,6(99,4)$ & 0,57 \\
\hline & Rho $=0,57, p<0,001$ & $X 2(3)=1,1 e+04, p<0,001$ \\
\hline
\end{tabular}

Fuente: Elaboración propia basada en antecedentes PSU-Demre 2013 y Simce 2011 


\subsection{Modelos explicativos}

En esta sección se presenta una serie de modelos de regresión logística ${ }^{30}$ que incluye las variables independientes de este estudio como predictores de la decisión de postular al sistema único de admisión universitario. Los predictores se incorporan de forma progresiva y en bloque, siguiendo una lógica paso a paso. El Modelo 1 incorpora las variables académicas, el Modelo 2 las variables relativas al origen socioeconómico y el Modelo 3 incluye la variable expectativas educativas. Posteriormente, los Modelos 4, 5 y 6 analizan la relación entre estas distintas variables. El Modelo 6 corresponde al modelo completo. La Tabla 4 presenta los resultados de estas distintas estimaciones.

Los resultados del Modelo 1 revelan que las variables académicas analizadas en este estudio -notas de enseñanza media, el puntaje ranking asociado y puntajes promedio PSU- predicen la decisión de postular al sistema único de admisión universitario. Mejores rendimientos en la enseñanza secundaria y, especialmente, puntajes más altos en las pruebas de selección universitaria, incrementan significativamente las probabilidades de los jóvenes de postular al sistema de admisión. Con el fin de cuantificar las probabilidades de postulación se desarrolló una serie de análisis complementarios sobre la base del Modelo 1. Estas probabilidades de postulación se calcularon considerando los puntajes PSU, definidos en deciles de puntajes. Los resultados revelaron que jóvenes con puntajes en el decil más alto de la distribución (decil 10) tienen un 90\% de probabilidades de postular al sistema universitario, mientras que aquellos que obtienen puntajes iguales o levemente superiores al puntaje mínimo de postulación (decil 5) solo tienen un 40\% de probabilidades de postular al sistema.

En los Modelos 4 y 6 se observa que en presencia de los controles socioeconómicos y expectativas educativas, la influencia

30 No se entregan estadísticos de ajuste de los modelos en la Tabla 4, ya que los modelos fueron calculados sobre la base de distintas bases de datos con datos imputados. Procedimientos estadísticos de posestimación o ajuste, no tienen una clara interpretación en modelos con datos imputados. 
de las variables académicas en la decisión de postulación persiste. Estos resultados vienen a confirmar la centralidad de los aspectos académicos en la decisión de postulación al sistema de admisión único universitario.

Por otra parte, los resultados también dan cuenta de diferencias significativas en las posibilidades de postulación al sistema universitario de acuerdo con el origen socioeconómico de los postulantes. Como se observa en el Modelo 2, los postulantes cuyos padres no tienen educación universitaria y desempeñan ocupaciones no profesionales (o de servicios) tienen menores probabilidades de postular al sistema de admisión único universitario que aquellos con padres universitarios y/o en ocupaciones profesionales.

Al controlar por las variables académicas del Modelo 4, se observa que las diferencias socioeconómicas disminuyen significativamente. Lo anterior, confirma lo planteado por la literatura acerca de la estrecha asociación entre el origen social y preparación académica de los postulantes (Breen \& Goldthorpe, 1997; Donoso y Cancino, 2007; Erikson \& Jonsson, 1996; Hernández y Paredes, 2007; Manzi, 2006; Muñoz y Redondo, 2013; Rodríguez, 2012). Los resultados sugieren que las diferencias socioeconómicas en probabilidades de postulación se deben, en buena medida, a los resultados académicos de los postulantes. Aquellos de orígenes socioeconómicos más aventajados tienen, en promedio, mejores rendimientos en las pruebas de selección obligatoria y durante la enseñanza media. Sus mejores rendimientos académicos explican, en gran parte, sus mayores posibilidades de postular al sistema de admisión universitario respecto de los jóvenes de orígenes socioeconómicos menos aventajados.

La educación parental, especialmente la del padre del postulante, se confirma como el factor socioeconómico más influyente en la decisión de postulación al sistema de admisión universitario. Se debe destacar que padres con educación universitaria influyen de manera positiva en la decisión de postulación de los jóvenes. Estos resultados podrían interpretarse de varias formas. Por una parte, pueden comprenderse como una clara señal de involucramiento parental en el proceso decisional que involucra la postulación 
al sistema de admisión universitario. Dado su conocimiento y experiencia en el sistema, los padres con educación universitaria pueden entregar a sus hijos asistencia, información y/o guía en el proceso que define la postulación al sistema de admisión (Hossler \& Stage, 1992; Stanton-Salazar \& Dornbusch, 1995). Por otra parte, estos resultados podrían estar revelando que la educación de los padres es también un referente importante para las propias elecciones educativas de los postulantes (Van de Werfhorst, Sullivan \& Cheung, 2003). Padres con educación universitaria influyen en que sus hijos decidan postular al sistema universitario, ya que sirven de modelos o roles a seguir.

Los resultados del Modelo 4 entregan evidencia para apoyar la hipótesis de efectos secundarios del origen social. Como se observa, al controlar por las variables académicas, las diferencias socioeconómicas en probabilidades de postulación persisten. Estas diferencias ${ }^{31}$, sugieren que jóvenes de distintos orígenes socioeconómicos, pero similar rendimiento académico difieren en la evaluación que realizan de sus habilidades y posibilidades de éxito en la postulación al sistema de admisión universitario. En la evaluación que los jóvenes hacen de sus habilidades, el origen social del postulante parece también influir de forma significativa. Lo anterior explicaría por qué jóvenes de orígenes sociales más aventajados deciden postular en mayor proporción al sistema de admisión universitario que postulantes de origen socioeconómico menos acomodado (Breen \& Goldthorpe 1997; Erikson \& Jonsson 1996; Jackson, et al., 2007).

Con el objeto de cuantificar las diferencias socioeconómicas en probabilidades de postulación al sistema de admisión universitario, se reestimó el Modelo $4^{32}$, incorporando una interacción entre el nivel educacional del padre y el puntaje del postulante en las pruebas de selección universitaria (medido en deciles de puntaje) ${ }^{33}$. Estos análisis

31 En el caso de los postulantes con padres sin estudios (o primaria incompleta), las diferencias en probabilidades de postulación desaparecen, luego de controlar por las variables académicas. Para este grupo de postulantes, las diferencias en probabilidades de postulación se deben exclusivamente a razones académicas.

32 El modelo controla por el resto de las variables académicas y socioeconómicas utilizadas en este estudio. Todas las variables fueron centradas en su media.

33 Los modelos no se presentan en este documento, pero pueden ser solicitados al autor. 
permitieron comparar las probabilidades de postulación de jóvenes que obtuvieron puntajes similares en la PSU y cuyos padres tienen distinto nivel educacional. A partir de estos análisis, se generaron probabilidades predichas, las que se presentan en el Gráfico 1. Como se observa allí, las probabilidades de postulación entre postulantes que obtienen puntajes similares en la PSU difieren de acuerdo con el nivel educativo de sus padres. En todos los tramos de puntaje, se observan diferencias socioeconómicas en las probabilidades de postulación. El Gráfico 1 muestra que, a similar puntaje PSU los postulantes cuyo padre tiene estudios universitarios siempre tienen mayores posibilidades de postulación que aquellos cuyo padre no tiene estudios universitarios. Resulta interesante destacar que las mayores diferencias socioeconómicas se concentran entre quienes obtienen puntajes medios y bajos en las pruebas de selección obligatoria; mientras que en los deciles más altos de puntajes, las diferencias socioeconómicas tienden a desaparecer. Estos resultados sugieren que postulantes de distintos orígenes socioeconómicos no procesan de la misma forma los bajos resultados en las pruebas de selección universitaria, lo cual apoya la tesis de las teorías de acción racional relativas a los efectos secundarios del origen social. 
Gráfico 1. Probabilidad de postular al sistema de admisión universitario, según decil de puntaje PSU y educación del padre del postulante

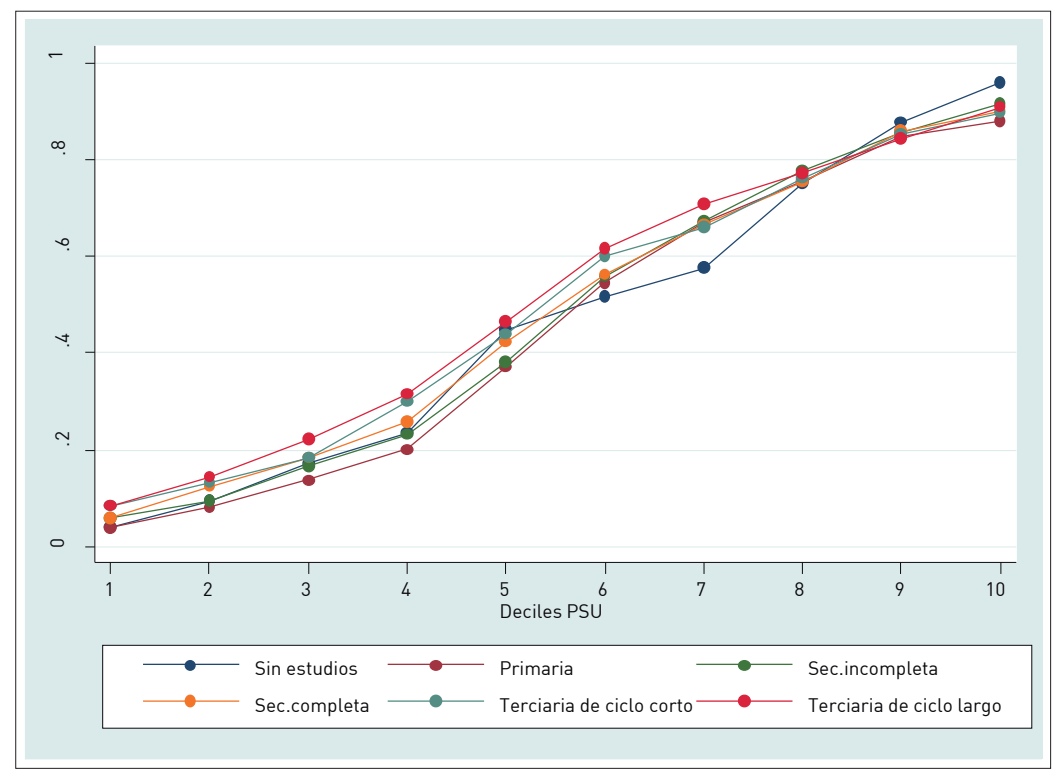

Finalmente, en relación con el rol de las expectativas educativas, los resultados confirman que estas son un factor que influye en la decisión de postular al sistema de admisión universitario. Como se observa en el Modelo 3, postulantes con proyecciones o aspiraciones universitarias previas son quienes tienen mayores posibilidades de postular al sistema de admisión. Adicionalmente, se observa que existe una significativa asociación entre expectativas educativas y desempeños académicos de los postulantes. Confirmando lo planteado por la literatura, el Modelo 6 revela que las expectativas educativas de los postulantes están mediadas por sus desempeños académicos (Andrew \& Hauser, 2012; Buchman \& Park 2009; Jerrim et al., 2015; Morgan, 2012; OECD, 2010, 2012). Jóvenes que tienen mayores expectativas educativas (o expectativas universitarias) tienen, en promedio, mejores desempeños académicos. Asimismo, los hallazgos de este estudio revelaron que solo parte de las diferencias en las aspiraciones educativas de los postulantes, se explican por sus rendimientos académicos. 
En relación con el vínculo entre expectativas educativas y origen socioeconómico, se observa que existe una relación significativa entre ambas variables; aunque esta relación es débil. Como muestra el Modelo 6, parte de las diferencias socioeconómicas en la decisión de postular al sistema de admisión se vincula con las aspiraciones educacionales previas de los postulantes. Específicamente, los resultados revelan que las expectativas educativas están asociadas con la educación de los padres del postulante. Análisis complementarios revelaron que los postulantes cuyos padres tienen menores niveles educativos -educación secundaria incompleta o menos- tienen expectativas más bajas que sus pares con padres más educados. Aspiraciones educativas más bajas (no universitarias) parecen explicar, en alguna medida, las menores posibilidades de postulación de postulantes de orígenes socioeconómicos menos aventajados. Estos resultados sugieren que la influencia del origen social en la decisión de postulación estaría también mediada por las aspiraciones previas de los postulantes y sus familias en torno a su futuro educacional y/u ocupacional.

Tabla 4. Modelos de regresión logística que predicen la probabilidad de postular al sistema de admisión universitario

\begin{tabular}{l|c|l|c|c|c|c}
\hline & Modelo 1 & Modelo 2 & Modelo 3 & Modelo 4 & Modelo 5 & Modelo 6 \\
\hline Puntaje notas enseñanza media & $0,007^{* * *}$ & & & $0,007^{* * *}$ & & $0,006^{* * *}$ \\
\hline & $(0,00)$ & & & $(0,00)$ & & $(0,00)$ \\
\hline Puntaje ranking & $-0,002^{* * *}$ & & & $-0,002^{* * *}$ & & $-0,002^{* * *}$ \\
\hline & $(0,00)$ & & & $(0,00)$ & & $(0,00)$ \\
\hline Puntaje promedio PSU & $0,02^{* * *}$ & & & $0,02^{* * *}$ & & $0,02^{* * *}$ \\
\hline & $(0,00)$ & & & $(0,00)$ & & $(0,00)$ \\
\hline
\end{tabular}

Educación del padre (ref.: Ed. Terciaria de ciclo largo)

\begin{tabular}{l|c|c|c|c|c|c}
\hline Sin estudios & & $-0,70^{* * *}$ & & $-0,11$ & $-0,64^{* * *}$ & $-0,09$ \\
\hline Ed. primaria & & $(0,07)$ & & $(0,09)$ & $(0,08)$ & $(0,09)$ \\
\hline & & $-0,64^{* * *}$ & & $-0,20^{* * *}$ & $-0,57^{* * *}$ & $-0,18^{* * *}$ \\
\hline Ed. secundaria incompleta & & $(0,02)$ & & $(0,03)$ & $(0,02)$ & $(0,03)$ \\
\hline & & $-0,53^{* * *}$ & & $-0,11^{* * *}$ & $-0,48 * *$ & $-0,10 * *$ \\
\hline Ed. secundaria completa & & $(0,02)$ & & $(0,03)$ & $(0,02)$ & $(0,03)$ \\
\hline & & $-0,42^{* * *}$ & & $-0,08 * *$ & $-0,39 * * *$ & $-0,07 * *$ \\
\hline Ed. terciaria ciclo corto & $(0,02)$ & & $(0,02)$ & $(0,02)$ & $(0,02)$ \\
\hline & & $-0,23^{* * *}$ & & $-0,05 *$ & $-0,22^{* * *}$ & $-0,05+$ \\
\hline & & $(0,02)$ & & $(0,03)$ & $(0,02)$ & $(0,03)$ \\
\hline
\end{tabular}




\begin{tabular}{|c|c|c|c|c|c|c|}
\hline \multicolumn{7}{|c|}{ Educación de la madre (ref.: ed. terciaria de ciclo largo) } \\
\hline \multirow[t]{2}{*}{ Sin estudios } & & $-0,85 * * *$ & & $-0,11$ & $-0,70^{* * *}$ & $-0,07$ \\
\hline & & $(0,08)$ & & $(0,1)$ & $(0,09)$ & $(0,1)$ \\
\hline \multirow[t]{2}{*}{ Ed. primaria } & & $-0,73^{* * *}$ & & $-0,14^{* * *}$ & $-0,64^{* * *}$ & $-0,12^{* * *}$ \\
\hline & & $(0,02)$ & & $(0,03)$ & $(0,02)$ & $(0,03)$ \\
\hline \multirow[t]{2}{*}{ Ed. secundaria incompleta } & & $-0,62^{* * *}$ & & $-0,05 *$ & $-0,56^{* * *}$ & $-0,04$ \\
\hline & & $(0,02)$ & & $(0,03)$ & $(0,02)$ & $(0,03)$ \\
\hline \multirow[t]{2}{*}{ Ed. secundaria completa } & & $-0,37^{* * *}$ & & 0,01 & $-0,34^{* * *}$ & 0,02 \\
\hline & & $(0,02)$ & & $(0,02)$ & $(0,02)$ & $(0,02)$ \\
\hline \multirow[t]{2}{*}{ Ed. terciaria ciclo corto } & & $-0,18^{* * *}$ & & $0,07 * *$ & $-0,17 * *$ & $0,07 * *$ \\
\hline & & $(0,02)$ & & $(0,03)$ & $(0,02)$ & $(0,03)$ \\
\hline \multicolumn{7}{|c|}{ Clase social del padre (ref.: clase de servicios) } \\
\hline \multirow[t]{2}{*}{ Clase de rutina no manual } & & 0,02 & & 0,03 & 0,01 & 0,03 \\
\hline & & $(0,02)$ & & $(0,02)$ & $(0,02)$ & $(0,02)$ \\
\hline \multirow[t]{2}{*}{ Pequeña burguesía } & & $0,08^{* *}$ & & $0,06^{*}$ & $0,05^{*}$ & $0,06+$ \\
\hline & & $(0,02)$ & & $(0,03)$ & $(0,03)$ & $(0,04)$ \\
\hline \multirow{2}{*}{ Trabajadores independiente } & & $-0,003$ & & $-0,08+$ & $-0,04$ & $-0,08+$ \\
\hline & & $(0,03)$ & & $(0,04)$ & $(0,04)$ & $(0,04)$ \\
\hline \multirow[t]{2}{*}{ Trabajadores manuales calificados } & & $-0,04+$ & & $0,07 * *$ & $-0,03$ & $0,08^{* *}$ \\
\hline & & $(0,02)$ & & $(0,02)$ & $(0,02)$ & $(0,02)$ \\
\hline \multirow[t]{2}{*}{ Trabajadores manuales no calificados } & & $-0,1^{* * *}$ & & $-0,02$ & $-0,09 * * *$ & $-0,01$ \\
\hline & & $(0,02)$ & & $(0,02)$ & $(0,02)$ & $(0,02)$ \\
\hline \multirow[t]{2}{*}{ Pequeños propietarios agrícolas } & & $0,13^{*}$ & & $0,16+$ & 0,11 & $0,14+$ \\
\hline & & $(0,07)$ & & $(0,09)$ & $(0,07)$ & $(0,08)$ \\
\hline \multirow[t]{2}{*}{ Trabajadores agrícolas } & & $-0,11 * * *$ & & 0,02 & $-0,07 * *$ & 0,04 \\
\hline & & $(0,03)$ & & $(0,03)$ & $(0,03)$ & $(0,03)$ \\
\hline \multicolumn{7}{|c|}{ Expectativa educativa (ref.: carrera universitaria) } \\
\hline \multirow[t]{2}{*}{ No cree que va a completar 4 to medio } & & & $-2,17 * * *$ & & $-2,11 * * *$ & $-1,09 * *$ \\
\hline & & & $(0,17)$ & & $(0,17)$ & $(0,21)$ \\
\hline \multirow[t]{2}{*}{ Cuarto medio } & & & $-2,21 * * *$ & & $-2,13^{* * *}$ & $-1,18^{* * *}$ \\
\hline & & & $(0,05)$ & & $(0,05)$ & $(0,05)$ \\
\hline \multirow[t]{2}{*}{$\begin{array}{l}\text { Carrera inst. prof. o centro de form. } \\
\text { técnica }\end{array}$} & & & $-1,57^{* * *}$ & & $-1,51 * * *$ & $-0,88^{* * *}$ \\
\hline & & & $(0,0)$ & & $(0,02)$ & $(0,02)$ \\
\hline $\mathrm{N}$ & 228,630 & 241,774 & 230,875 & 228,630 & 230,875 & 228,630 \\
\hline \multirow[t]{2}{*}{ Constante } & $11,56^{* * *}$ & $1,80^{* * *}$ & $1,82^{* * *}$ & $-10,49 * * *$ & $2,02^{* * *}$ & $-10,81 * * *$ \\
\hline & $(0,08)$ & $(0,04)$ & $(0,04)$ & $(0,08)$ & $(0,08)$ & $(0,09)$ \\
\hline
\end{tabular}

Todos los modelos controlan por edad, sexo, dependencia del establecimiento y modalidad de estudios cursada en educación. Los coeficientes son log odds, con errores estándares entre paréntesis*** $p<0,001{ }^{* *} p<0,01,{ }^{*} p<0,05,+p 0,1$ 


\section{Conclusiones}

Los resultados de este estudio confirman la centralidad de los factores académicos en la decisión de postular al sistema de admisión universitario chileno. La postulación al sistema de admisión es dependiente, en buena medida, de los desempeños o resultados académicos de los postulantes. Mejores puntajes en las pruebas de selección universitaria permiten a los jóvenes postular a las carreras de su preferencia, o alternativamente, elegir entre un pool amplio de opciones educativas, las carreras que se acerquen más a sus preferencias o intereses. Por otra parte, se constata la estrecha relación entre origen socioeconómico y resultados académicos de los postulantes. Las diferencias en resultados académicos explican en buena medida las diferencias socioeconómicas en la postulación al sistema de admisión universitario (Valdivieso et al., 2006).

Junto con lo anterior, se encontró que la influencia del origen socioeconómico en la postulación al sistema de admisión universitario no solo se relaciona con los resultados académicos de los postulantes. El origen social parece influir también directamente en su decisión de postulación al sistema. Los hallazgos sugieren que jóvenes de distinto origen social realizan una evaluación diferenciada de sus capacidades académicas y probabilidades de éxito a la hora de postular al sistema universitario. Para postulantes que provienen de grupos sociales más aventajados, sus resultados en las pruebas de selección universitaria parecieran ser menos determinantes en su decisión de postular al sistema, que para aquellos que provienen de orígenes menos aventajados. Por su parte, jóvenes de orígenes sociales más aventajados, con puntajes bajos y/o medios en las pruebas PSU, parecen sobreestimar sus opciones de éxito en su postulación, lo que los lleva a postular en mayor proporción que jóvenes de grupos menos aventajados al sistema de admisión universitario.

De las tres medidas de origen socioeconómico analizadas, la educación del padre del postulante resultó ser el factor socioeconómico más influyente en la decisión de postulación. La centralidad de la educación del padre en la decisión de postulación resulta un hallazgo novedoso. La investigación educacional chilena ha planteado que 
la educación de la madre es mucho más relevante que la educación del padre en los logros educativos de los estudiantes (Santos, 2009). Se presume que los resultados de este estudio difieren de esta literatura por dos razones: primero, porque este estudio analiza la educación superior, mientras que la literatura nacional ha analizado principalmente la educación escolar y, en segundo lugar, porque este estudio se centra en una decisión educativa, mientras que el foco de la literatura nacional ha estado puesto en los logros educativos.

Los resultados indican que padres con educación universitaria influyen positivamente en la postulación al sistema universitario. A modo de hipótesis, es posible plantear que su influencia positiva estaría relacionada con la entrega de información o guía a sus hijos en el proceso que define la postulación al sistema de admisión. Padres con estudios universitarios parecen constituir un piso o referente sobre el cual los estudiantes definen sus elecciones educativas (Mare \& Chang, 2006; Van de Werfhorst et al., 2003; Shavit et al., 2007).

Finalmente, en relación con las expectativas educativas de los postulantes, se encontró que estas median la decisión de postular al sistema universitario. Las diferencias socioeconómicas en la decisión de hacerlo se vinculan, en alguna medida, con las aspiraciones educacionales de los postulantes y sus familias. Se observa que las menores posibilidades de postulación de postulantes de origen socioeconómico bajo se deben, en parte, a aspiraciones no universitarias previas.

En síntesis, los resultados de este estudio confirman que la postulación al sistema de admisión universitario es un proceso en el que intervienen factores académicos y no académicos. Las desigualdades socioeconómicas en la postulación a la educación universitaria se relacionan principalmente con razones académicas, pero también con aspectos subjetivos de los postulantes. El origen socioeconómico de los postulantes parece influir en la forma en que los jóvenes evalúan sus capacidades académicas y visualizan sus posibilidades de éxito en el sistema universitario. 
154 DIFERENCIAS SOCIOECONÓMICAS EN LA POSTULACION A LAS UNIVERSIDADES

CHILENAS: EL ROL DE FACTORES ACADÉMICOS Y NO ACADÉMICOS - A. Canales

\section{Referencias}

Andrew, M. \& Hauser, R. M. (2012). Adoption? Adaptation? Evaluating the formation of educational expectations. Social Forces, 90(2), 497-520. http://dx.doi:10.1093/sf/sor005

Bernardi, F. \& Cebolla, H. (2013). Previous school results and social background: Compensation and imperfect information in educational transitions. European Sociological Review, 30(2), 207-217. http:// dx.doi:10.1093/esr/jct029

Boudon, R.(1974). Education, opportunity, and social inequality: changing prospects in western society. New York: Wiley-Interscience.

Bozick, R., Alexander, K., Entwisle, D., Dauber, S., \& Kerr, K. (2010). Framing the future: Revisiting the place of educational expectations in status attainment. Social Forces, 88(5), 2027-2052. http://dx.doi:10.1353/ sof.2010.0033

Breen, R. (1999). Beliefs, rational choice and bayesian learning. Rationality and Society, 11(4), 463-479. http://dx.doi:10.1177/104346399011004005

Breen, R. \& Goldthorpe, J. (1997). Explaining educational differentials: Towards a formal rational action theory. Rationality and Society, 9(3), 275-305. http://dx.doi:10.1177/104346397009003002

Buchmann, C. \& Park, H. (2009). Stratification and the formation of expectations in highly differentiated educational systems. Research in Social Stratification and Mobility, 27(4), 245-267. http://dx.doi. org/10.1016/j.rssm.2009.10.003

Castillo, J. y Cabezas, G. (2010). Caracterización de jóvenes primera generación en educación superior. Nuevas trayectorias hacia la equidad educativa. Calidad en la Educación, 32, 44-76.

Coleman, J. (1988). Social capital in the creation of human capital. American Journal of Sociology, 94(S), S95-S120. http://dx.doi:10.2307/2780243

Donoso, S. y Cancino, V. (2007). Caracterización socioeconómica de los estudiantes de educación superior. Calidad en la Educación, 26(1): 205-244.

Erikson, R. \& Jonsson, J. (1996). Can education be equalized? The Swedish case in comparative perspective. Colorado: Westview Press.

García Huidobro, J. E. y Bellei, C. (2003). Desigualdad educativa en Chile. En R. Hevia (Ed.), La educación en Chile hoy (pp. 1-63). Santiago de Chile: Universidad Diego Portales.

Hernández, L. y Paredes, R. (2007). Restricciones económicas en estudios técnicos o profesionales. Calidad en la Educación, 27(2), 238-261. 
Hossler, D. \& Stage, F. (1992). Family and high school experience influences on the postsecondary educational plans of ninth-grade students. American Educational Research Journal, 29(2), 425-451. http://dx.d oi:10.3102/00028312029002425

Jackson, M., Erikson, R., Goldthorpe, J., \& Meir, Y. (2007) Primary and secondary effects in class differentials in educational attainment: The transition to a-level courses in England and Wales. Acta Sociológica, 50(3), 211-229. http://dx.doi:10.1177/0001699307080926

Jerrim, J., Chmielewski, A., \& Parker, P. (2015). Socioeconomic inequality in access to high-status colleges: A cross-country comparison. Research in Social Stratification and Mobility, 42, 20-32. http://dx.doi.org/10.1016/j. rssm.2015.06.003

Jiménez, M., y Lagos, F. (2011) Nueva geografía de la educación superior y de los estudiantes: Una cartografía del sistema chileno, su actual alumnado y sus principales tendencias. Santiago de Chile: Universidad San Sebastián

Iatridis, T., \& Fousiani, K. (2009). Effects of status and outcome on attributions and just-world beliefs: how the social distribution of success and failure may be rationalized, Journal of Experimental Social Psychology, 45(2), 415-420

Lareau, A. \& Weininger, E. (2003). Cultural capital in educational research: A critical assessment. Theory and Society, 32(5/6), 567-606. http:// dx.doi:10.2307/3649652

Manzi, J. (2006) El acceso segmentado a la educación superior en Chile. En P. Díaz-Romero (Ed.), Caminos para la inclusión en la educación superior en Chile (pp. 187-204). Santiago de Chile: Equitas.

Mare, R. \& Chang, H. (2006). Family attainment norms and educational stratification in the United States and Taiwan: The effect of parents' school transitions. In G. Fields, D. Grusky, \& S. Morgan (Eds.), Frontiers in economic and social mobility (pp. 195-231) Stanford: Stanford University Press.

McDonough, P. (1997). Choosing colleges: How social class and schools structure opportunity. New York: State University of New York Press.

Ministerio de Educación, Mineduc. (2012). Realidad educativa en Chile: ¿Qué aprendemos de la Encuesta CASEN 2012? Santiago de Chile: Ministerio de Educación. Recuperado de http://centroestudios.mineduc.cl/tp_ enlaces/portales/tp5996f8b7cm96/uploadImg/File/A10N1_Casen.pdf

Morgan, S. (2002). Modeling preparatory commitment and non-repeatable decisions: Information processing, preference formation and 
educational attainment. Rationality and Society, 14(4), 387-429. http:// dx.doi:10.1177/1043463102014004001

Morgan, S., Leenman, T., Todd, J., \& Weeden, K. (2013). Occupational plans, beliefs about educational requirements, and patterns of college entry. Sociology of Education, 86(3), 197-217. http:// dx.doi:10.1177/0038040712456559

Muñoz, P. y Redondo, A. (2013). Desigualdad y logro académico en Chile. Revista Cepal, 119, 107-122.

Organisation for Economic Co-operation and Development, OECD. (2010). PISA 2009 results: Overcoming social background. Equity in learning opportunities and outcomes. Paris: OECD.

Organisation for Economic Co-operation and Development, OECD (2012). Grade expectations: How marks and education policies shape students' ambitions. Paris: OECD.

Reynolds, J. \& Johnson, M.K. (2011). Change in the stratification of educational expectations and their realization. Social Forces, 90(1), 85-109. http://dx.doi:10.1093/sf/90.1.85

Rodríguez, E. (2012). La educación superior en Chile y el rol del mercado: ¿culpable o inocente? Ingeniare. Revista Chilena de Ingeniería, 20(1), 126-135.

Roksa, J., Grodsky, E., Arum, R., \& Gamoran, A. (2007). The United States: Changes in higher education and social stratification. En Y. Shavit, R. Arum, A. Gamoran, \& G. Menahem (Eds.), Stratification in higher education: A comparative study (pp. 165-191). California: Stanford University Press.

Santos, H. (2009). Dinámica de la deserción escolar en Chile. Santiago de Chile: Centro de Políticas Comparadas de Educación, Universidad Diego Portales.

Sepúlveda, L. y Valdebenito, M. J. (2014). ¿Las cosas claras? Aspiraciones de futuro y proyecto educativo laboral de jóvenes estudiantes secundarios. Estudios Pedagógicos, 40(1), 243-261.

Sewell, W., Haller, A., \& Portes, A. (1969). The educational and early occupational attainment process. American Sociological Review, 34(1), 82-92. http://dx.doi:10.2307/2092789

Shavit, Y., Arum, R., Gamoran, A., \& Menahem, G. (2007). Stratification in higher education: A comparative study. California: Stanford University Press.

Silva, M. y Koljatic, M. (2010). Algunas reflexiones a siete años de la implementación de la PSU. Revista de Estudios Públicos, 120, 125-146. 
Stanton-Salazar, R. \& Dornbusch, S. (1995). Social capital and the reproduction of inequality: Information networks among Mexicanorigin high school students. Sociology of Education, 68(2), 116-135. http://dx.doi:10.2307/2112778

Torche, F. (2005). Unequal but fluid: Social mobility in Chile in comparative perspective. American Sociological Review, 70(3), 422-450. http:// dx.doi:10.2307/4145389

Valdivieso, P., Antivilo, A., y Barrios, J. (2006). Caracterización educacional y sociodemográfica de estudiantes que rinden la PSU, postulan y se matriculan en universidades del Consejo de Rectores. Revista Calidad en la Educación, 24(1), 319-361.

Van de Werfhorst, H. G., Sullivan, A., \& Cheung, S. Y. (2003). Social class, ability and choice of subject in secondary and tertiary education in Britain. British Educational Research Journal, 29(1), 41-62. http://dx.d oi:10.1080/0141192032000057366

Recibido: 14/10/2015

Aceptado: 06/05/2016 